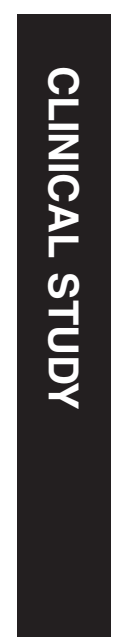

K-A Park and SY Oh

\section{Retinal nerve fiber layer thickness in prematurity is correlated with stage of retinopathy of prematurity}

Department of

Ophthalmology, Samsung

Medical Center,

Sungkyunkwan University

School of Medicine, Seoul,

Republic of Korea

Correspondence: SY Oh, Department of Ophthalmology, Samsung Medical Center,

Sungkynkwan University School of Medicine, Ilwondong, Kangnam-gu, Seoul, Republic of Korea Tel: +82 234103566 ; Fax: +82234100074 . E-mail: syoh@skku.edu

Received: 31 August 2014 Accepted in revised form: 29 June 2015

Published online: 25 September 2015

\begin{abstract}
Purpose To compare retinal nerve fiber layer (RNFL) thickness profiles between preterm and full-term children and to investigate factors affecting the RNFL distribution in preterm children.
\end{abstract}

Methods We performed Spectral domain optical coherence tomography (SD-OCT) peripapillary RNFL circular scan centered on the optic disc in $\mathbf{5 0}$ premature and $\mathbf{5 8}$ full-term children. RNFL thickness profiles were compared between preterm and fullterm children using a linear regression model. Among preterm patients in this study, 20 patients previously received laser treatment for severe retinopathy of prematurity (ROP).

Results Global average, nasal, and superior disc RNFL thickness profiles were significantly smaller in preterm children $(92.70 \pm 16.57 \mu \mathrm{m}, 56.02 \pm 17.04 \mu \mathrm{m}$, and $108.74 \pm 27.36 \mu \mathrm{m}$, respectively) compared with full-term children $(101.63 \pm 9.21 \mu \mathrm{m}, P=0.006,69.14 \pm 14.15 \mu \mathrm{m}$, $P<0.001$, and superior, $129.11 \pm 18.14 \mu \mathrm{m}$, $P<0.001$, respectively). Multivariable analysis revealed that ROP stage was inversely correlated with nasal RNFL thickness $(P=0.010)$.

Conclusions Our SD-OCT data demonstrate decreased global average, nasal, and superior disc RNFL thicknesses in preterm children. ROP stage was inversely correlated with nasal RNFL thickness. Further studies are needed to better understand the association between these structural changes and visual functions in preterm children.

Eye (2015) 29, 1594-1602; doi:10.1038/eye.2015.166; published online 25 September 2015

\section{Introduction}

Preterm birth immensely changes the infant's environment, which could lead to disruption of normal ocular development and function. The most common ocular complication associated with preterm birth is a vascular disorder called retinopathy of prematurity (ROP), which is known to cause enormous ocular complications associated with neovascularization. Recent studies using optical coherence tomography (OCT) have revealed that preterm children also have a thinner peripapillary retinal nerve fiber layer (RNFL) than children born at term, suggesting an association between prematurity and subclinical optic nerve hypoplasia. ${ }^{1,2}$ These studies showed a significant correlation between RNFL thickness and gestational age ${ }^{1}$ and birth weight. $^{2}$ Because gestational age and birth weight are closely related with each other and are also strongly correlated with development of other disorders including retinal vascular disorder, ROP, it is difficult to determine which factor is a critical contributor for the abnormality in the optic nerve development in prematurity. Ákerblom $e t a l^{3}$ recently attempted to determine the most critical factor involved in the abnormality of RNFL thickness and reported that ROP stage is inversely correlated with RNFL thickness. It was the first report to demonstrate that ROP stage is related with peripapillary RNFL thickness using spectral domain OCT (SD-OCT). However, this analysis was performed only for global average RNFL thickness, and whether this result came from a direct effect of ROP itself or was a sequel of laser treatment could not be determined. Laser treatment has been reported to induce 
peripapillary RNFL thinning, 4,5 and most of the subjects in their study with severe ROP had previously received laser treatment.

In our study, we compared RNFL thickness profiles between preterm and healthy, full-term children and determined the most important factor among gestational age, birth weight, and the stage of ROP in the peripapillary RNFL distribution in prematurity. Analysis of RNFL thickness profiles was performed separately depending on different sectors. We then discussed regarding implications and significance of our results.

\section{Materials and methods}

This prospective study was performed at a single center according to the tenets of the Declaration of Helsinki. The study was approved by our institutional review board. Written informed consent was obtained from the parents of the subjects before enrollment.

Our study involved two groups of children aged 4-14-year old: 53 preterm children and 60 healthy, full-term children, who visited the pediatric ophthalmology clinic for routine ocular examinations between the period 1 October 2011 and 31 August 2012. Inclusion criterion for preterm children was birth before 35 weeks gestational age. All preterm children had previously received ROP screening starting at 5 weeks after birth, which was repeated at least every other week. ROP screening continued until the retina was fully vascularized or for children with ROP, until ROP had resolved completely. The criterion for the treatment of ROP was stage 3 disease in at least four contiguous clock hours, even in the absence of plus disease. Inclusion criteria for full-term children included birth at term and normal birth weight. Some of these children had participated in the study of retinal and choroidal thickness in preterm children in the same department. ${ }^{6}$ Only children of age 4 years or older were included. Exclusion criteria included a history of stage 4 or 5 ROP, previous eye trauma, previous eye surgery, or inability to co-operate with OCT examination. Only right eyes were included in the study. We classified stages 1-2 ROP as mild and stage 3 ROP as severe, even in children who received laser treatment.

All subjects underwent full ophthalmologic assessments, including visual acuity testing, cycloplegic refraction, slit-lamp biomicroscopy, and fundus examination. Refractions were performed using retinoscopy after instillation of $1 \%$ cyclopentolate and $0.5 \%$ tropicamide. OCT cross-hair scans were performed with a SD-OCT (Spectralis, Heidelberg Engineering $\mathrm{GmbH}$, Heidelberg, Germany), which provided 40000 A-scans per second with $7 \mu \mathrm{m}$ optical and $3.5 \mu \mathrm{m}$ digital axial resolution. We obtained SD-OCT peripapillary
RNFL circular scans centered on the optic disc of each patient; the highest-quality image was selected for analysis (Figure 1). An internal fixation target was used, and the patient's opposite eye was covered during scanning.

Statistical analyses were performed using SAS (version 9.2). RNFL thickness profiles were compared between preterm and full-term children using a linear regression model adjusting for the patient's spherical equivalent refractive error and the patient's age at the time of examination. Associations between RNFL thickness and multiple factors including gestational age at birth, birth weight, and ROP stage were analyzed using both Pearson's correlation analysis and linear regression model. Correlations between visual acuity and multiple factors including gestational age at birth, birth weight, ROP stage, and RNFL thickness profiles were analyzed using Spearman's correlation analysis. A median regression model was also used for multivariable analysis. Owing to multiple testing, the resultant $P$-values were corrected using Bonferroni correction. Student's $t$-test was used to compare gestational age at birth, birth weight, age at examination, spherical equivalent refractive error, and visual acuity between preterm and full-term children. Pearson's $\chi^{2}$-test was used to compare gender between the two groups.

\section{Results}

Of the 53 enrolled preterm children, 50 were used for the analysis. Of the 60 enrolled full-term children, 58 were used for the analysis of retinal layer thickness and choroidal thickness. Three preterm and two full-term children were excluded from the study owing to poor scan image quality. Baseline characteristics of the children including demographics, gestational age at birth, birth weight, age at testing, refractive error (described by spherical equivalent), history of ROP, ROP stage, and laser treatment for ROP are shown in Table 1. The mean refractive error of preterm children was $-1.03 \pm 3.22$, which was slightly more myopic than full-term children among whom the mean refractive error was $-0.54 \pm 1.69$ $(P=0.477)$.

Table 2 compares peripapillary RNFL thickness profiles between preterm and full-term children. Significant differences were observed between preterm and full-term children in terms of peripapillary RNFL thickness. The average total, nasal, and superior peripapaillry RNFL thicknesses were significantly smaller in preterm children (average total, 92.70 \pm 16.57 , nasal, $56.02 \pm 17.04 \mu \mathrm{m}$, and superior, $108.74 \pm 27.36 \mu \mathrm{m}$ ) compared with full-term children (average total, 101.63 $\pm 9.21, P=0.006$, nasal, $69.14 \pm 14.15 \mu \mathrm{m}, P<0.001$, and superior, $129.11 \pm 18.14 \mu \mathrm{m}, P<0.001)$. 

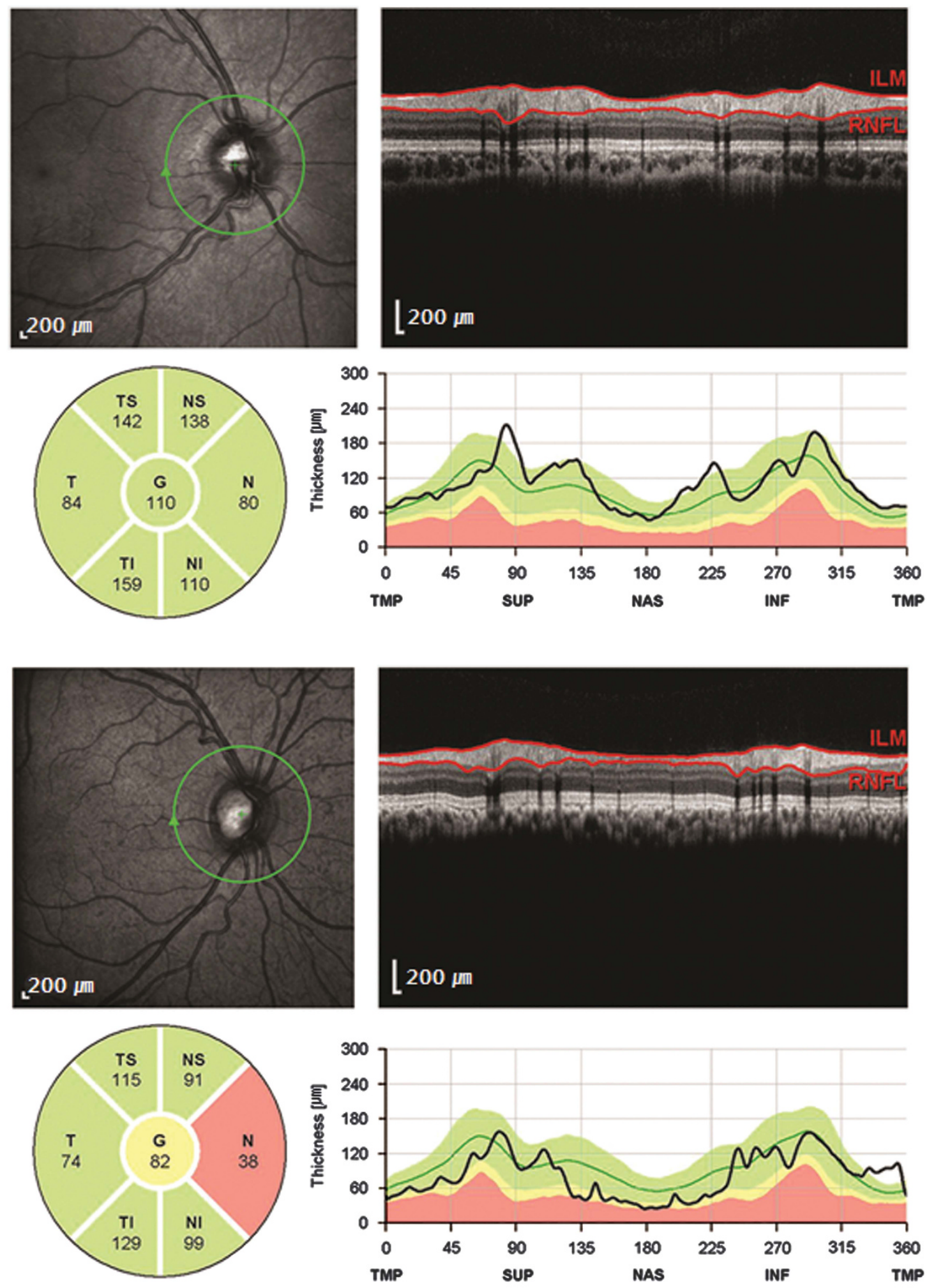

Figure 1 Representative peripapillary retinal nerve fiber layer (RNFL) thickness profiles from a full-term control child (top) and a preterm child (bottom). Each half of the figure includes a fundus photograph that illustrates the location of the peripapillary RNFL circular scan, the spectral domain optical coherence tomography B-scan, a pie chart that provides the calculated average thickness for each of the four sectors centered on the optic disc and the global average thickness, and an RNFL thickness profile. 
Pearson's correlation analysis between baseline patient parameters and peripapillary RNFL thicknesses revealed that gestational age was inversely correlated with global average and temporal peipapillary RNFL thicknesses ( $r=-0.44, P=0.008$ and $r=-0.38, P=0.035)$; birth weight was inversely correlated with temporal RNFL thickness ( $r=-0.40, P=0.021)$; ROP stage was positively correlated with the average total and temporal RNFL thicknesses

Table 1 Demographic and clinical description of the preterm children and children born at term

\begin{tabular}{lccc}
\hline Variable & $\begin{array}{c}\text { Preterm } \\
(\mathrm{n}=50) \\
\text { Mean } \pm S D\end{array}$ & $\begin{array}{c}\text { Full-term } \\
(\mathrm{n}=58) \\
\text { Mean } \pm S D\end{array}$ & P-value \\
\hline Gestational age at birth & $28.08 \pm 3.23$ & $\geq 37.00$ & \\
(weeks) & $1.18 \pm 0.45$ & $\geq 2.50$ & \\
Birth weight (kg) & $6.88 \pm 2.29$ & $7.50 \pm 2.98$ & $0.079^{\mathrm{a}}$ \\
Age at testing (years) & $25 / 25$ & $26 / 32$ & $0.697^{\mathrm{b}}$ \\
Gender (male/female) & $-1.03 \pm 3.22$ & $-0.54 \pm 1.69$ & 0.477 \\
Refractive error (diopters) & 34 & & \\
Retinopathy of & & & \\
prematurity (n) & 11 & & \\
$\quad$ Mild (stages 1-2) & 23 & & \\
Severe (stage 3) & 20 & & \\
Laser treatment (n) & & & \\
Periventricular hemorrhage and periventricular leukomalacia & \\
$\quad$ Periventricular hemorrhage & 2 & & \\
$\quad$ Periventricular leukomalacia & 6 & & \\
\hline at-test. ${ }^{b}$ Pearson's $\chi^{2}$-test. &
\end{tabular}

Table 2 Descriptive statistics (mean \pm SD) and statistical comparisons of peripapillary retinal nerve fiber layer thickness in preterm children and children born at term adjusted by age at examination and spherical equivalent refractive error

\begin{tabular}{lccr}
\hline Variable & $\begin{array}{c}\text { Preterm }(n=50) \\
\text { Mean } \pm S D\end{array}$ & $\begin{array}{c}\text { Full-term }(n=58) \\
\text { Mean } \pm S D\end{array}$ & P-value $^{a}$ \\
\hline Global average & $92.70 \pm 16.57$ & $101.63 \pm 9.21$ & 0.006 \\
Temporal & $88.84 \pm 25.54$ & $80.34 \pm 11.43$ & 1.000 \\
Nasal & $56.02 \pm 17.04$ & $69.14 \pm 14.15$ & $<0.001$ \\
Superior & $108.74 \pm 27.36$ & $129.11 \pm 18.14$ & $<0.001$ \\
Inferior & $122.60 \pm 24.44$ & $127.20 \pm 15.22$ & 1.000
\end{tabular}

Abbreviations: SD, standard deviation; RNFL, retinal nerve fiber layer. a $t$-test.
( $r=0.41, P=0.014$ and $r=0.55, P<0.001)$. Multivariable analysis revealed that ROP stage was inversely correlated with nasal RNFL thickness $(P=0.010)$ (Table 3) (Figure 2). Gestational age and birth weight were not determined as significant factors affecting RNFL thickness in multivariable analysis.

LogMAR visual acuity was significantly worse in preterm children $(0.14 \pm 0.20)$ compared with full-term children $(0.02 \pm 0.03, P<0.001)$. Pearson's correlation and multivariable analyses revealed that visual acuity in preterm children was not correlated with RNFL thicknesses, gestational age at birth, birth weight, and ROP stage (Table 4).

\section{Discussion}

Our analysis of peripapillary RNFL thickness showed a significantly thinner global average RNFL thickness in preterm children compared with full-term children. This result is in agreement with recent studies using SD-OCT. ${ }^{1,2,7}$ With regard to sectoral distributions of peripapillary RNFL, it has been reported that peripapillary RNFL thickness on the temporal side of the disc is thicker in preterm children than in full-term controls, whereas all other peripapillary RNFL sectors are thinner in preterm children than those in full-term children. ${ }^{1,2}$ In this study, the results also showed that all peripapillary RNFL sectors except for the temporal side were thinner in preterm children than those in full-term controls. Statistically significant differences were observed in the average total, nasal, and superior disc RNFL thicknesses after adjustment for patients' age at examination and spherical equivalent refractive error. Univariate analysis revealed significant inverse correlations between peripapillary RNFL thicknesses, and gestational age and birth weight in the temporal sector. Gestational age and birth weight were not significantly correlated with RNFL thickness in sectors other than the temporal side. The stage of ROP was positively correlated with the average global and temporal peripapillary RNFL thicknesses in the

Table 3 Association of baseline characteristics with peripapillary retinal nerve fiber layer in preterm patients (a multivariable model)

\begin{tabular}{|c|c|c|c|c|c|c|c|c|c|c|}
\hline \multirow[t]{3}{*}{ Variable } & \multicolumn{10}{|c|}{ Retinal nerve fiber layer thicknesses } \\
\hline & \multicolumn{2}{|c|}{ Average total } & \multicolumn{2}{|c|}{ Temporal } & \multicolumn{2}{|c|}{ Nasal } & \multicolumn{2}{|c|}{ Superior } & \multicolumn{2}{|c|}{ Inferior } \\
\hline & Estimate & P-value $e^{\mathrm{a}}$ & Estimate & P-value ${ }^{\mathrm{a}}$ & Estimate & P-value ${ }^{\mathrm{a}}$ & Estimate & P-value $e^{\mathrm{a}}$ & Estimate & P-value ${ }^{\mathrm{a}}$ \\
\hline Gestational age at birth & -1.51 & 1.000 & -2.28 & 1.000 & -0.21 & 1.000 & -1.59 & 1.000 & -2.94 & 0.491 \\
\hline Birth weight & 0.01 & 1.000 & 0.25 & 1.000 & -9.94 & 1.000 & 7.22 & 1.000 & 8.84 & 1.000 \\
\hline ROP stage & -8.49 & 0.800 & -2.78 & 1.000 & -26.3 & 0.010 & 5.88 & 1.000 & -12.69 & 0.705 \\
\hline Age at examination & -1.01 & 1.000 & -1.48 & 1.000 & -2.13 & 0.718 & 0.20 & 1.000 & -0.51 & 1.000 \\
\hline SE refractive error & 0.51 & 1.000 & 1.15 & 1.000 & -2.76 & 0.049 & 1.21 & 0.843 & 2.03 & 1.000 \\
\hline
\end{tabular}

Abbreviations: ROP, retinopathy of prematurity; SE, spherical equivalent. ${ }^{\text {a }}$ Mixed model adjusting patient's spherical equivalent refractive error and age at the examination. 


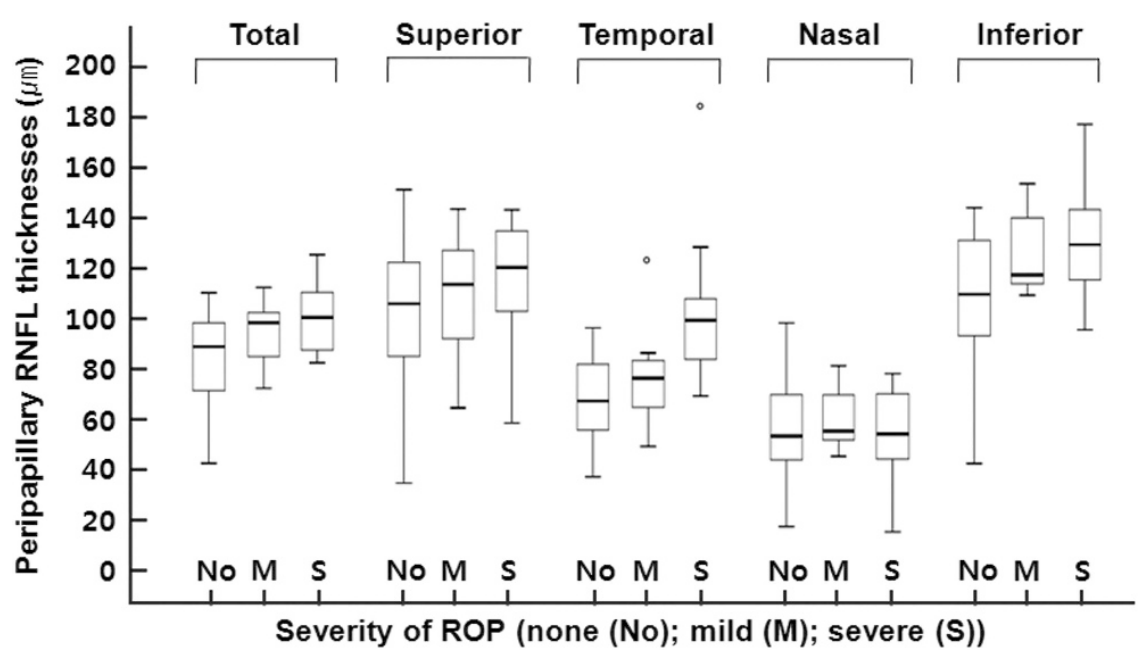

Figure 2 Boxplot demonstrating the correlation between the stage of retinopathy of prematurity and LogMAR visual acuity in preterm patients. Central bars indicate median values and boxes indicate interquartile ranges, with whiskers extending to the highest and lowest values. Excluded are the values that extend $>3 / 2$ of the interquartile range from the edge of the box. Open circles indicate values beyond this point. RNFL, retinal nerve fiber layer; ROP, retinopathy of prematurity.

Table 4 Association of baseline characteristics with LogMAR visual acuity at examination in preterm patients (a multivariable model)

\begin{tabular}{|c|c|c|c|c|c|c|c|c|c|c|}
\hline \multirow[t]{2}{*}{ Variable } & \multicolumn{10}{|c|}{ LogMAR visual acuity } \\
\hline & Estimate & P-value $e^{\mathrm{a}}$ & Estimate & P-value $e^{\mathrm{a}}$ & Estimate & P-value ${ }^{\mathrm{a}}$ & Estimate & P-value ${ }^{\mathrm{a}}$ & Estimate & P-value \\
\hline Gestational age at birth & 0.01 & 0.389 & 0.01 & 0.315 & 0.01 & 0.405 & 0.01 & 0.616 & 0.02 & 0.311 \\
\hline Birth weight & -0.10 & 0.367 & -0.12 & 0.211 & -0.08 & 0.456 & -0.06 & 0.575 & -0.11 & 0.312 \\
\hline ROP stage & -0.03 & 0.711 & 0.11 & 0.153 & -0.01 & 0.898 & -0.04 & 0.551 & -0.03 & 0.696 \\
\hline \multicolumn{11}{|l|}{ RNFL thickness } \\
\hline Global average & -0.00 & 0.609 & & & & & & & & \\
\hline Temporal & & & 0.00 & 0.171 & & & & & & \\
\hline Nasal & & & & & 0.00 & 0.623 & & & & \\
\hline Superior & & & & & & & -0.00 & 0.430 & & \\
\hline Inferior & & & & & & & & & -0.00 & 0.557 \\
\hline Age at examination & -0.03 & 0.021 & -0.03 & 0.012 & 0.01 & 0.015 & -0.03 & 0.016 & -0.03 & 0.018 \\
\hline Presence of anisometropia & 0.01 & 0.873 & -0.02 & 0.747 & 0.07 & 0.744 & 0.01 & 0.905 & 0.00 & 1.000 \\
\hline Presence of strabismus & 0.03 & 0.644 & 0.02 & 0.614 & 0.06 & 0.695 & 0.03 & 0.682 & 0.04 & 0.560 \\
\hline
\end{tabular}

Abbreviations: ROP, retinopathy of prematurity; RNFL, retinal nerve fiber layer. ${ }^{a}$ Mixed model adjusting patient's spherical equivalent refractive error and age at the examination.

univariate analysis. Interestingly, although statistically significant differences in RNFL thicknesses between preterm and full-term children were observed in the average global RNFL thickness and in sectors including superior and nasal sides; the largest thinning of the mean RNFL thickness in preterm children was observed in the nasal sector, statistically significant correlations between baseline factors and RNFL thickness was mainly observed in RNFL thicknesses in the temporal sector.

Wang et al ${ }^{1,8}$ hypothesized that such abnormalities in the spatial profile of RNFL would be present based on abnormal inner retinal layers overlaying the fovea in preterm children. Recent studies have revealed that preterm children have thicker central maculae than children born at term, regardless of the presence of ROP. ${ }^{6,9,10}$ Another recent study has shown that premature birth is associated with the failure of multiple retinal layers to migrate away from the fovea, resulting in increased foveal thickness in patients with ROP. ${ }^{8}$ This immature and not fully migrated retinal layers could directly contribute to the increased thickness of peripapillary RNFL thicknesses in the temporal sector. It is also possible that this temporal thickening and thinning of other sectors in preterm children compared 
with full-term controls are reflections of immature development of neurovascular structures around the optic disc itself. As Wang et al ${ }^{11}$ previously pointed out, those distributions represent a less-arcuate distribution of the peripapillary RNFL, considering the fact that the temporal sector of the RNFL is normally the thinnest portion of the normal RNFL profile.

Multivariable analysis also revealed that ROP stage was inversely correlated with nasal RNFL thickness. Gestational age and birth weight were not significant factors affecting RNFL thickness in multivariable analysis. Thinning of the RNFL in the superior and nasal side might be simply related to disruption of normal development owing to preterm birth. However, it is also possible that some sort of pathologic process related to the development of ROP may contribute to typical distributions of RNFL in preterm children. Åkerblom et al also showed that the presence of severe ROP was related to the thinning of the global average RNFL. ${ }^{3}$ During the development of ROP, the relatively vasculardepleted retina induces overproduction of hormones and growth factors, including vascular endothelial growth factor and insulin-like growth factor- $1 .^{12}$ These factors influence proteins of the extracellular matrix to deposit adhesive fibrins and induce growth, differentiation and migration of endothelial cells. ${ }^{13}$ Numerous factors participate in the genesis of ROP including low birth weight, getational age, and high postnatal oxygenation. Preterm infants who suffer complications of prematurity exhibit a total absence of autoregulation of ocular blood flow resulting in an exaggerated delivery of potentially toxic oxygen to the retina when they are exposed to oxygen supplementation or overcome respirator insufficiency. ${ }^{14}$ A rise in carbon dioxide tension is another factor, which significantly contributes to disruption in the regulation of retinal and choroidal blood flow in preterm infants. ${ }^{15,16}$ Sharing of these background phenomena not only induces the development of ROP in preterm children but may also interfere with normal maturation of the optic disc.

However, it is unclear if ROP affects RNFL thickness directly, or the development of ROP and thinning of RNFL in the nasal sector just shares same background properties. As Åkerblom et $a l^{3}$ pointed out earlier, ROP itself might affect RNFL thinning, and laser treatment for ROP could induce RNFL thinning. As RNFL thickness around the optic nerve has been reported to be thinned with progression of diabetic retinopathy, ${ }^{4}$ we can postulate that severe ROP could damage the axons of ganglion cells in a similar manner. In addition, majority of our study patients with severe ROP had received laser treatment, which could also lead to RNFL thinning. It is well documented that laser photocoagulation induces reduction of peripapillary RNFL thickness. ${ }^{5,17}$ Because the number of children with previous severe ROP without laser treatment was too small to allow proper sub-analyses, it was impossible to clearly reveal whether laser treatment had an effect on RNFL thickness in this study. However, in univariate analysis, the presence of severe ROP showed a significant positive correlation with RNFL thickness in the temporal sector. Patients with severe ROP had a thicker RNFL in the temporal sector compared with normal full-term controls. The effect of laser treatment on RNFL thickness alone cannot fully explain this result. Thus, we can postulate that other pathologic mechanisms including various factors described above, in addition to the effect of laser treatment, probably have some role in the development of characteristic features of RNFL distribution observed in preterm children. Periventricular hemorrhage, which is more frequently found in very low birth weight infants has been reported to be associated with reduced optic disc diameter, optic disc area, and optic cup area. ${ }^{18} \mathrm{We}$ could not include the presence of periventricular hemorrhage in the multivariable analysis because there were only two patients who had periventricular hemorrhage. We hope further studies will clearly reveal the association between peripapillary RNFL distribution and the presence of severe intraventricular hemorrhage. Another possible explanation for the thinning of the RNFL in the sectors except for the temporal sector is artifacts from the vessels. It has been reported that children with low birth weight have narrower arterioles, ${ }^{19}$ and arteriolar caliber is positively correlated with RNFL thickness and macular thickness. ${ }^{20-22}$ Therefore, narrower vessels in preterm children compared with normal full-term controls could induce artifactually thinned RNFL measured by OCT. ${ }^{23}$ Further histologic studies could help to clarify this idea.

In this study, LogMAR visual acuity was significantly worse in preterm children compared with full-term children. Pearson's correlation analysis and multivariable analysis failed to find significant factors including RNFL thicknesses, gestational age at birth, birth weight, and ROP stage associated with poor visual acuity, except for the age at examination. Because these factors have complicated relations with each other, it might be hard to find significant factor for the poor visual acuity in preterm patients. Although recent studies showed that retinal layer thickness at the fovea was not associated with visual acuity in preterm children, 8,24,25 Wang et $a l^{1}$ reported that peripapillary RNFL thickness on the temporal side of the disc is related with visual acuity. Peripapillary RNFL is closely correlated with multiple visual deficits including visual field defect, low-contrast sensitivity, and abnormal 
forms of visual function tests, including contrast sensitivity, visual filed test, electrophysiology test, could more clearly elucidate the association between visual functions and structural abnormalities in the peripapillary region of preterm children. did not perform tests for visual functions other than visual acuity, we could not determine whether typical features in the peripapillary RNFL distribution observed in preterm patients is related to deficits in other visual functions. Regarding the problem of compliance in young children, further studies involving older children who can co-operate with other thorough visual function tests are needed.

The main strength of this study is that we adjusted refractive error and age at examination in every analysis. Refractive error and age at examination are some of the well-known confounding factors in the interpretation of peripapillary RNFL thickness. ${ }^{32-38}$ In addition, we performed correlation analysis between various baseline factors and peripapillary RNFL thicknesses in multiple sectors separately. As there was a difference in the pattern of RNFL distribution according to different sectors, it would be more accurate to analyze RNFL thickness in each sector separately. This study also has several limitations. Our study population was recruited from a single institution, and all of them were of the same race. We also could not completely rule out selection bias because we recruited children from a hospital, not from a community. Owing to the paucity of preterm children with a history of severe ROP who had not received previous laser treatment, we could not conclusively determine how much laser treatment itself affected the thinning of the RNFL in preterm children with severe ROP. However, patients with severe ROP showed a larger temporal RNFL thickness, suggesting that laser treatment, which is known to induce RNFL thinning, might not be the sole cause of the characteristic features of the peripapillary RNFL distribution in preterm children. At last, our inclusion criterion for full-term birth was birth from $\geq 37$ weeks. The full-term child included in this study who was born earliest was born at 37 weeks. Retinal development and inner retina might be different between patients born at 37 weeks and patients born at 42 weeks. In conclusion, this study confirmed previous findings on the structural characteristics of the peripapillary RNFL in preterm children and is the first to analyze the effect of ROP stage on RNFL thickness in different locations of the peripapillary region. The stage of ROP showed a positive correlation with the temporal RNFL thickness and an inverse correlation with nasal RNFL thickness. Further studies with larger participant populations including preterm children with a history of severe ROP without laser treatment may reveal the mechanism of contribution of ROP stage on peripapillary RNFL thickness. In addition, studies using different

\section{Summary}

What was known before

- Recent studies using OCT have revealed that preterm children also have a thinner peripapillary RNFL than children born at term, suggesting an association between prematurity and subclinical optic nerve hypoplasia. critical factor involved in the abnormality of RNFL thickness and reported that ROP stage is inversely correlated with RNFL thickness. It was the first report to demonstrate that ROP stage is related with peripapillary RNFL thickness using SD-OCT. However, this analysis was performed only for global average RNFL thickness, and whether this result came from a direct effect of ROP itself or was a sequel of laser treatment could not be determined. Laser treatment has been reported to induce peripapillary RNFL thinning, and most of the subjects in their study with severe ROP had previously received laser treatment.

\section{What this study adds}

- This study confirmed previous findings on the structural characteristics of the peripapillary RNFL in preterm children and is the first to analyze the effect of ROP stage on RNFL thickness in different locations of the peripapillary region. The stage of ROP showed a positive correlation with the temporal RNFL thickness and an inverse correlation with nasal RNFL thickness.

\section{Conflict of interest}

The authors declare no conflict of interest.

\section{Acknowledgements}

No financial support was provided for this study. The authors have completed and submitted the ICMJE form for disclosure of potential conflicts of interest and none were reported. Contribution of authors: Involved in conception and design (KAP); analysis and interpretation (KAP, SYO); writing the article (KAP, SYO); critical revision of the article (KAP, SYO); final approval of the article (KAP, SYO); data collection (KAP, SYO); provision of materials, patients, or resources (KAP, SYO); literature search (KAP); Administrative, technical, or logistic support (SYO). This study was supported by the Samsung Medical Center Clinical Research Development Program, grant \#CRS-111-07-1. Åkerblom et al recently attempted to determine the most 


\section{References}

1 Wang J, Spencer R, Leffler JN, Birch EE. Characteristics of peripapillary retinal nerve fiber layer in preterm children. Am J Ophthalmol 2012; 153: 850-855 e851.

2 Tariq YM, Pai A, Li H, Afsari S, Gole GA, Burlutsky G et al. Association of birth parameters with OCT measured macular and retinal nerve fiber layer thickness. Invest Ophthalmol Vis Sci 2011; 52: 1709-1715.

3 Akerblom H, Holmstrom G, Eriksson U, Larsson E. Retinal nerve fibre layer thickness in school-aged prematurely-born children compared to children born at term. Br J Ophthalmol 2012; 96: 956-960.

4 Park HY, Kim IT, Park CK. Early diabetic changes in the nerve fibre layer at the macula detected by spectral domain optical coherence tomography. Br J Ophthalmol 2011; 95: 1223-1228.

5 Lim MC, Tanimoto SA, Furlani BA, Lum B, Pinto LM, Eliason $\mathrm{D}$ et al. Effect of diabetic retinopathy and panretinal photocoagulation on retinal nerve fiber layer and optic nerve appearance. Arch Ophthalmol 2009; 127: 857-862.

6 Park KA, Oh SY. Analysis of spectral-domain optical coherence tomography in preterm children: retinal layer thickness and choroidal thickness profiles. Invest Ophthalmol Vis Sci 2012; 53: 7201-7207.

7 Wang XY, Huynh SC, Rochtchina E, Mitchell P. Influence of birth parameters on peripapillary nerve fiber layer and macular thickness in six-year-old children. Am J Ophthalmol 2006; 142: 505-507.

8 Wang J, Spencer R, Leffler JN, Birch EE. Critical period for foveal fine structure in children with regressed retinopathy of prematurity. Retina 2012; 32: 330-339.

9 Akerblom H, Larsson E, Eriksson U, Holmstrom G. Central macular thickness is correlated with gestational age at birth in prematurely born children. Br J Ophthalmol 2011; 95: 799-803.

10 Yanni SE, Wang J, Chan M, Carroll J, Farsiu S, Leffler JN et al. Foveal avascular zone and foveal pit formation after preterm birth. Br J Ophthalmol 2012; 96(7): 961-966.

11 Hood DC, Kardon RH. A framework for comparing structural and functional measures of glaucomatous damage. Prog Retin Eye Res 2007; 26: 688-710.

12 Rivera JC, Sapieha P, Joyal JS, Duhamel F, Shao Z, Sitaras N et al. Understanding retinopathy of prematurity: update on pathogenesis. Neonatology 2011; 100: 343-353.

13 Pollan C. Retinopathy of prematurity: an eye toward better outcomes. Neonatal Netw 2009; 28: 93-101.

14 Milligan DW. Failure of autoregulation and intraventricular haemorrhage in preterm infants. Lancet 1980; 1: 896-898.

15 Checchin D, Hou X, Hardy P, Abran D, Najarian T, Beauchamp MH et al. PGE(2)-mediated eNOS induction in prolonged hypercapnia. Invest Ophthalmol Vis Sci 2002; 43: 1558-1566.

16 Holmes JM, Zhang S, Leske DA, Lanier WL. Carbon dioxideinduced retinopathy in the neonatal rat. Curr Eye Res 1998; 17: 608-616.

17 Muqit MM, Wakely L, Stanga PE, Henson DB, Ghanchi FD. Effects of conventional argon panretinal laser photocoagulation on retinal nerve fibre layer and driving visual fields in diabetic retinopathy. Eye (Lond) 2010; 24: 1136-1142.

18 McLoone E, O'Keefe M, Donoghue V, McLoone S, Horgan N, Lanigan B. RetCam image analysis of optic disc morphology in premature infants and its relation to ischaemic brain injury. Br J Ophthalmol 2006; 90: 465-471.
19 Mitchell P, Liew G, Rochtchina E, Wang JJ, Robaei D, Cheung $\mathrm{N}$ et al. Evidence of arteriolar narrowing in lowbirth-weight children. Circulation 2008; 118: 518-524.

20 Cheung N, Tong L, Tikellis G, Saw SM, Mitchell P, Wang JJ et al. Relationship of retinal vascular caliber with optic disc diameter in children. Invest Ophthalmol Vis Sci 2007; 48: 4945-4948.

21 Lim LS, Saw SM, Cheung N, Mitchell P, Wong TY. Relationship of retinal vascular caliber with optic disc and macular structure. Am J Ophthalmol 2009; 148: 368-375.

22 Samarawickrama C, Huynh SC, Wang JJ, Pai A, Joachim N, Burlutsky $\mathrm{G}$ et al. Relationship between retinal structures and retinal vessel caliber in normal adolescents. Invest Ophthalmol Vis Sci 2009; 50: 5619-5624.

23 Hood DC, Fortune B, Arthur SN, Xing D, Salant JA, Ritch R et al. Blood vessel contributions to retinal nerve fiber layer thickness profiles measured with optical coherence tomography. J Glaucoma 2008; 17: 519-528.

24 Park KA, Oh SY. Analysis of spectral-domain optical coherence tomography in preterm children: retinal layer thickness and choroidal thickness profiles. Invest Ophthalmol Vis Sci 2012; 53(11): 7201-7207.

25 Recchia FM, Recchia CC. Foveal dysplasia evident by optical coherence tomography in patients with a history of retinopathy of prematurity. Retina 2007; 27: 1221-1226.

26 Arantes TE, Garcia CR, Tavares IM, Mello PA, Muccioli C. Relationship between retinal nerve fiber layer and visual field function in human immunodeficiency virus-infected patients without retinitis. Retina 2012; 32: 152-159.

27 Horn FK, Kaltwasser C, Junemann AG, Kremers J, Tornow RP. Objective perimetry using a four-channel multifocal VEP system: correlation with conventional perimetry and thickness of the retinal nerve fibre layer. $\mathrm{Br} J$ Ophthalmol 2012; 96: 554-559.

28 Kalyani PS, Holland GN, Fawzi AA, Arantes TE, Yu F, Sadun AA. Association between retinal nerve fiber layer thickness and abnormalities of vision in people with human immunodeficiency virus infection. Am J Ophthalmol 2012; 153: 734-742, 742 e731.

29 Leaney J, Healey PR, Lee M, Graham SL. Correlation of structural RNFL parameters and functional measures using HRT3 and Spectralis SD-OCT at different levels of glaucoma severity. Clin Experiment Ophthalmol 2012; 40(8): 802-812

30 Nilforushan N, Nassiri N, Moghimi S, Law SK, Giaconi J, Coleman AL et al. Structure-function relationships between spectral-domain OCT and standard achromatic perimetry. Invest Ophthalmol Vis Sci 2012; 53: 2740-2748.

31 Walter SD, Ishikawa H, Galetta KM, Sakai RE, Feller DJ, Henderson SB et al. Ganglion cell loss in relation to visual disability in multiple sclerosis. Ophthalmology 2012; 119: $1250-1257$.

32 Alasil T, Wang K, Keane PA, Lee H, Baniasadi N, de Boer JF et al. Analysis of normal retinal nerve fiber layer thickness by age, sex, and race using spectral domain optical coherence tomography. J Glaucoma 2013; 22(7): 532-541.

33 Bendschneider D, Tornow RP, Horn FK, Laemmer R, Roessler CW, Juenemann AG et al. Retinal nerve fiber layer thickness in normals measured by spectral domain OCT. J Glaucoma 2010; 19: 475-482.

34 Budenz DL, Anderson DR, Varma R, Schuman J, Cantor L, Savell $\mathrm{J}$ et al. Determinants of normal retinal nerve fiber layer thickness measured by Stratus OCT. Ophthalmology 2007; 114: 1046-1052. 
35 Hirasawa H, Tomidokoro A, Araie M, Konno S, Saito H, Iwase A et al. Peripapillary retinal nerve fiber layer thickness determined by spectral-domain optical coherence tomography in ophthalmologically normal eyes. Arch Ophthalmol 2010; 128: 1420-1426.

36 Parikh RS, Parikh SR, Sekhar GC, Prabakaran S, Babu JG, Thomas R. Normal age-related decay of retinal nerve fiber layer thickness. Ophthalmology 2007; 114: 921-926.
37 Schuman JS, Hee MR, Puliafito CA, Wong C, PedutKloizman T, Lin CP et al. Quantification of nerve fiber layer thickness in normal and glaucomatous eyes using optical coherence tomography. Arch Ophthalmol 1995; 113: 586-596.

38 Varma R, Bazzaz S, Lai M. Optical tomography-measured retinal nerve fiber layer thickness in normal latinos. Invest Ophthalmol Vis Sci 2003; 44: 3369-3373. 